\title{
Plasma phospholipase A2 activity may serve as a novel diagnostic biomarker for the diagnosis of breast cancer
}

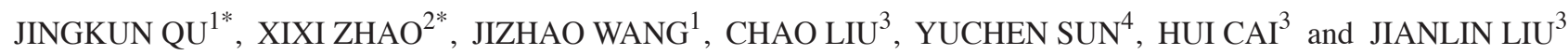 \\ ${ }^{1}$ The Second Department of Thoracic Surgery, The First Affiliated Hospital of Xi'an Jiaotong University, Xi'an, \\ Shaanxi 710061; ${ }^{2}$ Department of Oncology, The Second Affiliated Hospital of Xi'an Jiaotong University, \\ Xi'an, Shaanxi 710004; Departments of ${ }^{3}$ Vascular Surgery and ${ }^{4}$ Radiation Oncology, \\ The First Affiliated Hospital of Xi'an Jiaotong University, Xi'an, Shaanxi 710061, P.R. China
}

Received October 1, 2016; Accepted November 23, 2017

DOI: $10.3892 / \mathrm{ol} .2018 .7915$

\begin{abstract}
Previous studies have indicated that phospholipase A2 (PLA2) may be associated with tumorigenesis in human tissues. The present study aimed to investigate the association between plasma PLA2 activity and the breast cancer (BC) status of patients. Increased plasma PLA2 activity was detected in patients with breast cancer when compared with healthy controls. Plasma samples were obtained from patients with $\mathrm{BC}(\mathrm{n}=169)$, patients with benign disease $(\mathrm{BD} ; \mathrm{n}=80)$ and healthy controls $(n=81)$. PLA2 activity was assessed using a quantitative fluorescent assay with selective inhibitors. It was demonstrated that increased PLA2 and secretory PLA2 (sPLA2) activity was associated with tumor stage, particularly in patients with late-stage disease. Additionally, smoking, alcohol consumption, body mass index (BMI) and age of patients did not have a significant effect on PLA2 activity. Analysis of receiver operating characteristic curves revealed that plasma PLA2 and SPLA2 activities were increased in BC patients compared with healthy controls. It was concluded that plasma PLA2 activity may serve as a biomarker for patients with BC.
\end{abstract}

Correspondence to: Dr Hui Cai or Dr Jianlin Liu, Department of Vascular Surgery, The First Affiliated Hospital of Xi'an Jiaotong University, 277 West Yanta Road, Xi'an, Shaanxi 710061, P.R. China E-mail: caihui9@stu.xjtu.edu.cn

E-mail: jlliu@mail.xjtu.edu.cn

${ }^{*}$ Contributed equally

Abbreviations: BC, breast cancer; BD, benign disease; PLA2, phospholipase A2; sPLA2-IIa, group IIa secretory phospholipase A2; AA, arachidonic acid

Key words: phospholipase A2, secretory phospholipase A2, breast cancer, biomarker, plasma

\section{Introduction}

According to the American Cancer Society, breast cancer (BC) is the most common malignancy in women, accounting for $>246,660$ cases and 40,450 fatalities in America alone in 2016 (1). The incidence of BC is rising, particularly in high-income and developed countries (2). Several subtypes of $\mathrm{BC}$ have been identified, each exhibiting variations in clinical behavior. Previous research has led to improvements in the early diagnosis and treatment of BC, including developments in target therapy, which has prolonged the survival rate of patients (3). However, more effective therapeutic agents and novel biomarkers are required to improve the prognosis of patients with BC (4).

Previous studies have demonstrated that expression of phospholipase A2 (PLA2) is associated with the initiation and progression of certain types of malignant cancer, including gastrointestinal, colorectal and prostate carcinomas (5-8). PLA2 is the name given to enzymes that catalyze the deacylation of glycerophospholipids at the sn-2 position, producing two lipid mediators, fatty acid derivatives and lysophospholipids $(9,10)$, which serve as signaling molecules in various types of cancer. PLA2 has also been identified as a potential target of cancer therapy. It has been revealed that mammals possess $>30$ enzymes with PLA2 activity (9). PLA2 enzymes are classified into four groups based on differing molecular mechanisms, including cellular localization, substrate specificity and calcium dependence. These are: Cytosolic PLA2 (cPLA2), calcium-independent PLA2 (iPLA2), secreted PLA2 (sPLA2) and platelet activating factor acetyl hydrolases (11). It has been demonstrated that sPLA2-IIa, a secretory PLA2 enzyme, is highly expressed in certain human cells and tissues, and is associated with various diseases, including cancer $(5,12)$.

Plasma biomarkers can be assessed by low-cost, non-invasive methods, potentially enabling the early diagnosis of various diseases (13). The present study examined the activities of PLA2 and SPLA2 in blood plasma samples obtained from patients with $\mathrm{BC}$, patients with benign disease (BD) and healthy participants. The results of a validated, quantitative and reproducible PLA2 assay (14) utilized in the current study indicated that the activity of plasma PLA2 and SPLA2 in patients with BC were 
significantly higher than that in healthy participants. In addition, PLA 2 and SPLA 2 activities were associated with BC tumor stages. Other potential risk factors for BC development (15-17), including smoking, alcohol consumption, body-mass index (BMI) and age, were also assessed in the present study.

\section{Materials and methods}

Human sample collection and processing. Clinical plasma samples were collected from patients with BC $(\mathrm{N}=169)$, patients with $\mathrm{BD}(\mathrm{N}=80)$ and healthy participants $(\mathrm{N}=81)$ from the First Affiliated Hospital of Xi'an Jiaotong University (Shaanxi, China) between August 2013 and November 2015. The mean age was 53.25 years, with a range of 28 to 77 years old. BC and benign lesions were confirmed using immunohistochemical staining after ultrasound-guided breast biopsies. Healthy participants were recruited under the conditions of a free routine health examination. All participants in this study satisfied the following inclusion criteria: i) Proven diagnosis of breast cancer or benign disease by pathology; ii) no adjuvant therapy or surgery prior to blood sample collection from patients with breast cancer; iii) female. Exclusion criteria were as follows: i) Previous history of cancer, infectious disease or other health conditions, which may have altered the results of the present study; ii) absence of a complete medical record. Blood samples were collected in the morning on an empty stomach in the presence of EDTA and centrifuged at $3,000 \mathrm{x} \mathrm{g}$, at $4^{\circ} \mathrm{C}$ for $30 \mathrm{~min}$, aliquoted into siliconized Eppendorf tubes and stored at $-80^{\circ} \mathrm{C}$. The present study was reviewed and approved by the ethics committee of The First Affiliated Hospital of Xi'an University Jiaotong University (Shaanxi, China) and written informed consent was obtained from all patients prior to enrollment. All clinical investigations were conducted according to the principles detailed in the Declaration of Helsinki (18).

The histopathological staining procedure of the BC tissues obtained. Tissues were first deparaffinized for $45 \mathrm{sec}$ at $30^{\circ} \mathrm{C}$ in $100 \%$ xylene then fixed for $45 \mathrm{sec}$ at $30^{\circ} \mathrm{C}$ in $100 \%$ propanol. A single drop of $5 \%$ hematoxylin stain was applied per $50-100 \mathrm{~mm}^{2}$ tissue for $45 \mathrm{sec}$ at $30^{\circ} \mathrm{C}$. The slides were then rinsed vigorously with distilled water at $30^{\circ} \mathrm{C}$ for $45 \mathrm{sec}$ prior to 2-3 drops per slide of $1 \%$ eosin stain for $30 \mathrm{sec}$ at $30^{\circ} \mathrm{C}$. The slides were rinsed again with cold $4^{\circ} \mathrm{C}$ distilled water for $15 \mathrm{sec}$, prior to a final wash for $45 \mathrm{sec}$ in $100 \%$ propanol at $30^{\circ} \mathrm{C}$ and then $45 \mathrm{sec}$ in $100 \%$ xylene at $30^{\circ} \mathrm{C}$. A Leica DM4 B light microscope (at a $\mathrm{x} 400$ magnification) was used to visualize the staining.

Reagents and inhibitors. The fluorescent substrate of PLA2, 1-O-(6-Dabcyl-aminohexanoyl)-2-O-(12-(5-BODIPY-entanoyl)aminododecanoyl)-sn-glyceryl phosphatidylcholine (DBPC) was obtained from Echelon Biosciences, Inc. (Salt Lake City, UT, USA). The dual inhibitor of cPLA2 and iPLA2, methyl arachidonyl flourophosphonate (MAFP) was obtained from Santa Cruz Biotechnology Inc. (Dallas, TX, USA) (19).

Analyse of PLA2 and SPLA2 enzymatic activity. PLA2 activity was assessed using DBPC; a fluorogenic phosphatidylcholine substrate (14). Plasma samples $(0.1 \mathrm{ml})$ were mixed with DBPC
(0.2 mg with PBS) to a final volume of $200 \mathrm{ml}$ and concentration if $0.001 \mathrm{mg} / \mathrm{ml}$ ). Then put them in $37^{\circ} \mathrm{C}$ temperature incubator. The fluorescence of samples was determined using a Victor3 V plate reader, wavelength $515 \mathrm{~nm}$, (PerkinElmer Inc., Waltham, MA, USA) at 30 min intervals over $2 \mathrm{~h}$. Variations in PLA2 activities can be measured by measuring the change in fluorescence intensity/min $/ \mu$ l of plasma. The following conditions were selected to distinguish the activity of PLA2 from the aforementioned subtypes: Normal PLA2 activity was assessed without exogenous additives and SPLA2 activity was examined in the presence of $1.2 \mathrm{mM}$ calcium chloride (the natural ionized calcium concentration in blood) and MAFP (10 $\mu \mathrm{M}$; a dual inhibitor of cPLA2 and iPLA2).

Freeze-thaw cycling. PLA2 activity stability was analyzed as aforementioned both prior and subsequent to sample-storage at $-80^{\circ} \mathrm{C}$ for half a month. This is one Freeze-thaw cycle. The coefficient of variation $(\mathrm{CV})=$ standard deviation $/$ mean value.

Statistical analysis. Categorical variables were summarized as counts with percentages and continuous variables were summarized as the mean \pm standard deviation across the healthy control, BD and BC groups. Pearson's correlation coefficient was used to determine associations between two variables. A non-parametric test was used to evaluate the difference between the median values of two groups. A Kruskal-Wallis test and a post-hoc Nemenyi test were used to assess the median fluorescence intensity values between the three groups. One-way analysis of variance with post-hoc Student-Newman-Keuls test was applied to assess the association between enzyme activity and univariates, including smoking, alcohol drinking, BMI and age. Multivariate linear regression analysis was used to estimate the interaction between dependent variables and multiple independent variables, including group, smoking, second-hand smoking (when a patient lives in the household of a smoker). alcohol consumption, BMI and age. Stepping method criteria were used to adjust for confounding factors. Receiver operating characteristic (ROC) curve analysis was performed to test the diagnostic value of plasma PLA2 activities. All analyses were performed using SPSS 19.0 (IBM corp., Armonk, NY, USA). $\mathrm{P}<0.05$ was considered to indicate a statistically significant difference.

\section{Results}

Patient demographic data. All patients with BC were confirmed using histopathological staining and classified based on Tumor-Node-Metastasis staging (20). The mean ages of the healthy controls, patients with $\mathrm{BD}$ and patients with $\mathrm{BC}$ were $52.65 \pm 8.64,51.58 \pm 7.80$ and $54.34 \pm 9.78$ years, respectively. The demographic data of age, smoking status, alcohol consumption and BMI are presented in Table I.

PLA2 and SPLA2 activities are elevated in patients with $B C$. The activities of plasma PLA2 (without additives) and sPLA2 were analyzed using one-way analysis Multivariate linear regression analysis was used to estimate the interaction between dependent variables and multiple independent 
Table I. Patient demographic data.

\begin{tabular}{|c|c|c|c|c|}
\hline & Healthy controls ${ }^{\mathrm{a}}$ & Patients with $\mathrm{BD}^{\mathrm{b}}$ & Patients with $\mathrm{BC}^{\mathrm{c}}$ & P-value \\
\hline Smoking status & & & & 0.523 \\
\hline Current smoker, n (\%) & $8(9.9)$ & $6(7.5)$ & $20(11.8)$ & \\
\hline Past smoker, n (\%) & $8(9.9)$ & $7(8.8)$ & $17(10.1)$ & \\
\hline Never smoked, n (\%) & $65(80.2)$ & $67(83.8)$ & $132(78.1)$ & \\
\hline Alcohol consumption & & & & 0.339 \\
\hline Does not consume & $52(64.2)$ & $47(58.8)$ & $114(68.3)$ & \\
\hline Consumes & $29(35.8)$ & $33(41.3)$ & $53(31.7)$ & \\
\hline $\mathrm{BMI}^{\mathrm{d}}, \mathrm{kg} / \mathrm{m}^{2}$ & $24.04 \pm 3.60$ & $24.61 \pm 3.58$ & $24.98 \pm 3.47$ & 0.781 \\
\hline Age $^{\mathrm{d}}$, years & $52.65 \pm 8.64$ & $51.58 \pm 7.80$ & $54.34 \pm 9.78$ & 0.065 \\
\hline
\end{tabular}

variables. Stepping method criteria were used to adjust for confounding factors and the variables of smoking status, alcohol consumption, BMI and age were ruled out, leaving only the differing group variable. The adjusted $\mathrm{R}^{2}$ values of PLA2 and sPLA2 were 0.152 and 0.137 , respectively. Multiple comparisons revealed that PLA2 and sPLA2 activity were significantly increased in patients with $\mathrm{BC}(\mathrm{P}<0.001$; Fig. 1).

Reproducibility and stability of the PLA2 assays. DBPC-based PLA2 assays were utilized. A total of 10 representative plasma samples (randomly selected from each group) were analyzed 3 times and the coefficient of variation (CV) was adopted to measure the discrete degree of sample reproducibility. The average CV of PLA2 and SPLA2 was 1.28 and $2.54 \%$, respectively, indicating that this method possessed a good repeatability. The effect of a freeze-thaw cycle on the stability and activity of PLA2 was examined by comparing the results of fresh samples with those in samples stored at $-80^{\circ} \mathrm{C}$ for half a month. The average CV of PLA2 and SPLA2 was 0.79 and $2.86 \%$, respectively, as previously reported (19). The PLA2 activity assay demonstrated good reproducibility and stability, considering the minimal CV of repeatability and freeze-thaw for the PLA2 and sPLA2 results.

Clinical manifestations and PLA2 activities. PLA2 and sPLA2 activities were significantly increased in plasma samples obtained from patients with BC compared with patients with $\mathrm{BD}$ and with healthy participants $(\mathrm{P}<0.05)$. This suggests that PLA2 and sPLA2 maybe potential biomarkers for breast cancer. However, whether they were associated with cancer advancement remains unclear. To obtain the required statistics, the activities of PLA2 and sPLA2 in plasma samples from patients with different tumor stages were compared. The results demonstrated that natural PLA2 and SPLA2 activities were increased in patients with late-stage $\mathrm{BC}$ (stages III and IV; $\mathrm{P}<0.001$; Fig. 2). This indicated that the enzymatic activities of PLA2 and sPLA2 induced the progression of $\mathrm{BC}$.
Plasma PLA2 activities possess a good diagnostic value. To assess the diagnostic capabilities of PLA2 and sPLA2, an ROC curve analysis was performed using plasma samples obtained from patients with $\mathrm{BC}$ and healthy controls. It was revealed that PLA2 and SPLA2 exhibited area under the curve values of 0.783 and 0.748 , with sensitivities of 72 and $60.1 \%$ and specificities of 72 and $82 \%$, respectively (Fig. 3). These results indicate that PLA2 and SPLA2 may possess diagnostic values in discriminating between patients with $\mathrm{BC}$ and healthy individuals.

Impacts of other risk factors on PLA2 activity. Risk factors that may affect the activity of PLA2, including smoking, alcohol consumption, age and BMI, were analyzed in the present study. A total of 330 patients were assessed for smoking history: no smoking history, $80.2 \%$ (healthy controls), $83.8 \%$ (BD), $78.1 \%$ (BC); current smokers, $9.9 \%$ (healthy controls), $7.5 \%$ (BD), $11.8 \%$ (BC); previous smokers $9.9 \%$ (healthy controls), $8.8 \%$ (BD), $11.8 \%$ (BC). There was no difference among the 3 groups regarding smoking status and PLA2/sPLA2 ( $\mathrm{P}>0.05$; Fig. 4A and B).

Alcohol consumption was determined for a total of 328 patients. The percentage of patients that consumed alcohol in healthy control patients, patients with $\mathrm{BD}$ and patients with were $35.8,41.3$ and $31.7 \%$, respectively. There was no difference of PLA2/sPLA2 activity among the 3 groups comparing alcohol consumption with no alcohol consumption: healthy group, $\mathrm{P}=0.412$ (PLA2), $\mathrm{P}=0.375$ (sPLA2); $\mathrm{BD}$ group, $\mathrm{P}=0.805$ (PLA2), $\mathrm{P}=0.259$ (sPLA2); $\mathrm{BC}$ group, $\mathrm{P}=0.168$ (PLA2), $\mathrm{P}=0.291$ (sPLA2) (Fig. 5).

The body mass indexes (BMIs) of 324 patients were also determined, revealing an even distribution of BMI values, and no statistically significant difference of PLA2 and SPLA2 activities comparing patients of a $\mathrm{BMI} \leq 25$ with $\mathrm{BMI}>25$ : healthy group, $\mathrm{P}=0.348$ (PLA2), $\mathrm{P}=0.085$ (sPLA2); $\mathrm{BD}$ group, $\mathrm{P}=0.572$ (PLA2), $\mathrm{P}=0.865$ (sPLA2); $\mathrm{BC}$ group, $\mathrm{P}=0.414$ (PLA2), $\mathrm{P}=0.710$ (sPLA2) (Fig. 6).

Age is an established risk factor for $\mathrm{BC}$ as menopause may affect its development. To remove this confounding factor, 


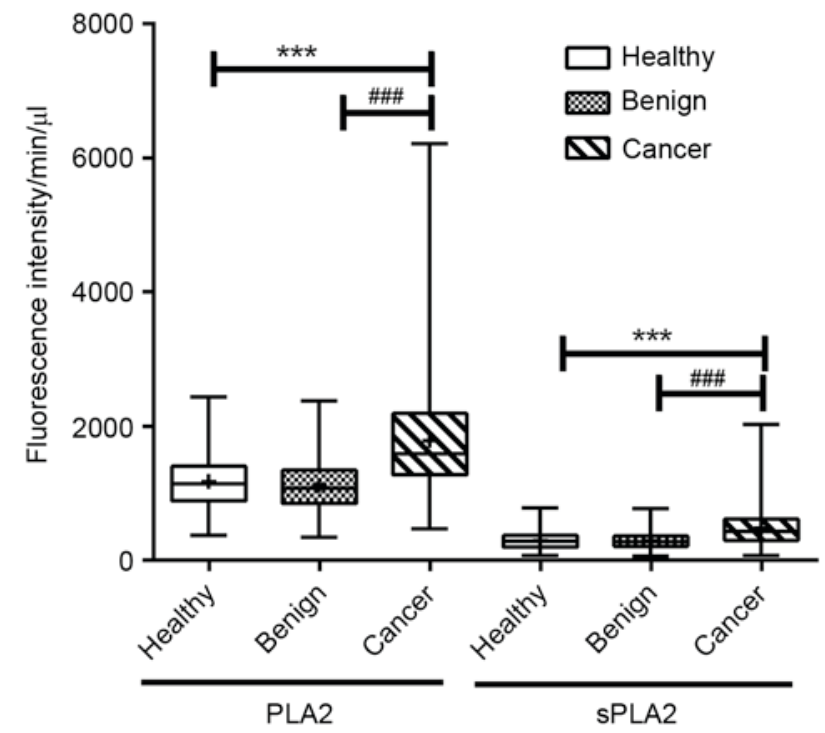

Figure 1. Plasma PLA2/sPLA2 activity was indicated by fluorescence intensity. Plasma PLA2/sPLA2 activity was elevated in patients with breast cancer. The activities of PLA2/sPLA2 were compared in the healthy controls $(\mathrm{n}=81)$, patients with benign disease $(\mathrm{n}=80)$ and patients with breast cancer $(\mathrm{n}=169)$. The distribution of natural PLA2/sPLA2 activity was analyzed as described in the Materials and methods. Statistically significant differences were identified between patients with breast cancer and healthy controls/patients with benign disease $\left({ }^{* * *} \mathrm{P}<0.001\right.$ healthy vs. cancer; ${ }^{\# \#} \mathrm{P}<0.001$ benign vs. cancer). PLA2, phospholipase A2; sPLA2, secretory phospholipase A2. ( $\mathrm{P}<0.05$ was considered to indicate a statistically significant difference). The box and whisker plots from top to bottom represent the maximum value, the upper quartile, the mean value, the lower quartile and the minimum value.

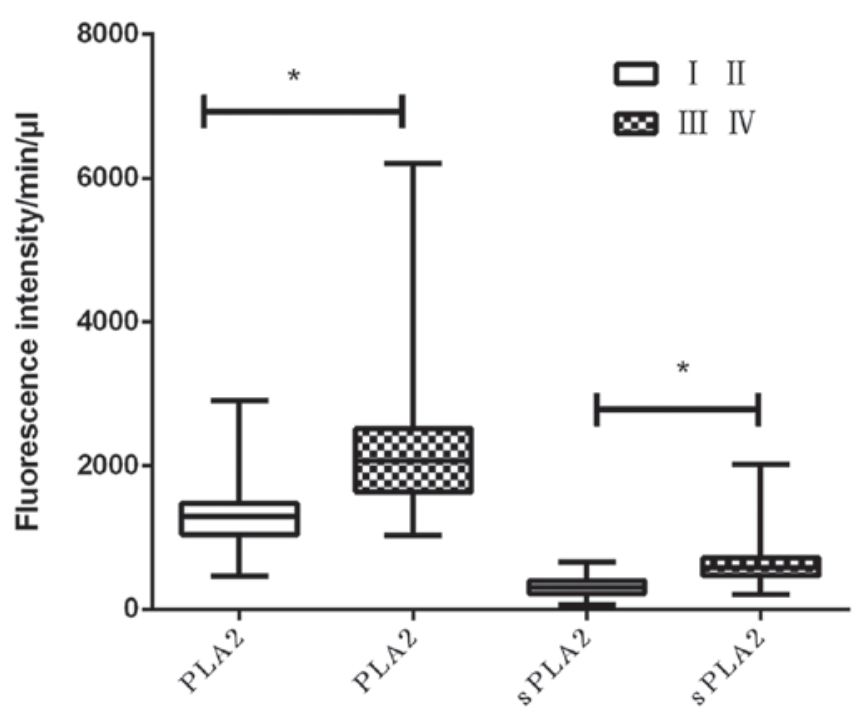

Figure 2. Plasma PLA2/sPLA2 activity is associated with T stage. PLA2 and sPLA2 activity was compared among patients with different $\mathrm{T}$ stages (TI and TII vs. TIII \& TIV). The results demonstrated that PLA2 and sPLA2 activity was increased in TIII \& TIV patients compared with TI and TII patients. PLA2, phospholipase A2; sPLA2, secretory phospholipase A2. "P<0.05 was considered to indicate a statistically significant difference.

patients in $\mathrm{BC}, \mathrm{BD}$ and healthy controls were then divided into two subgroups: Those aged $<50$ and those aged $\geq 50$ years PLA2 and sPLA2 remained unchanged in two age subgroups among BC, BD and healthy controls (Fig. 7).

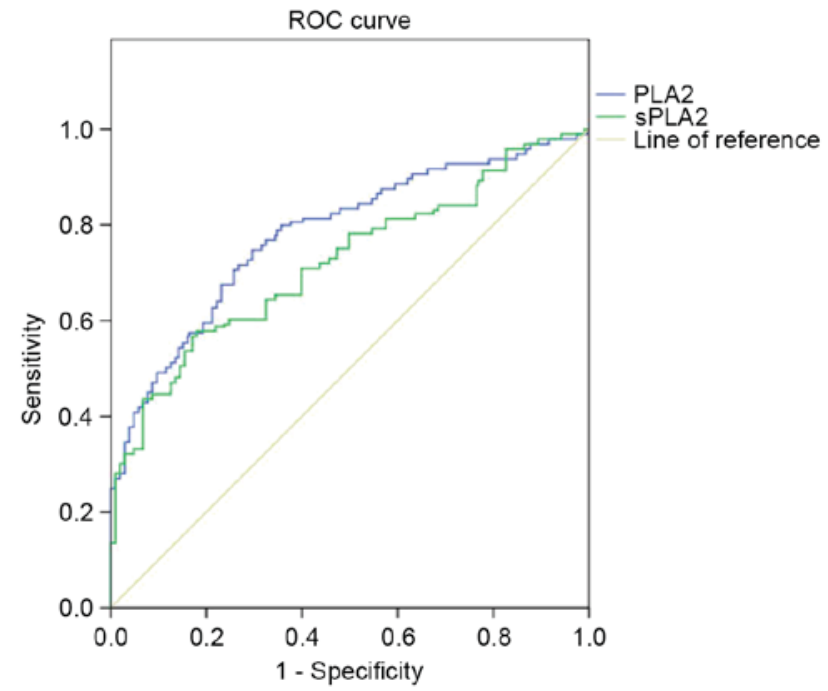

Figure 3. Plasma PLA2 and sPLA2 activities possess a good diagnostic value. Plasma PLA2 and SPLA2 activity in patients with breast cancer produced an area under the curve value of 0.783 and 0.748 , respectively. These results demonstrated that PLA2 and sPLA2 activity possess a considerable diagnostic ability to discriminate between breast cancer patients and healthy controls. PLA2, phospholipase A2; sPLA2, secretory phospholipase A2; ROC, receiver operating characteristic curve.

\section{Discussion}

The present study determined that the activities of PLA2 and sPLA2 in plasma may serve as novel biomarkers for patients with BC. One drew similar conclusions regarding colorectal cancer, lung cancer, bladder cancer and pancreas cancer (19). The present study assessed PLA2 obtained without additives, so that the activity of PLA2 was considered 'natural'. Optimal conditions, i.e. in the presence of $1.2 \mathrm{mM}$ calcium chloride (the natural ionized calcium concentration in blood) and $10 \mathrm{mM}$ MAFP were also selected for the assessment of sPLA2 activity.

The present study assessed the confounding factors that are associated with PLA2 activity. In the present study, it was determined that patient age, BMI, smoking status or alcohol consumption did not differ significantly between the three groups of patients assessed. In addition, Buhmeida et al (21) demonstrated that there were no significant associations between PLA2 expression and age, sex, depth of invasion or lymph node status. Furthermore, Yamashita et al (22) did not identify an association between steroid hormone receptor status and the concentration of PLA2 in BC tissues. These results were also consistent with those produced by Mannello et al (12). However, induced abortion, oral contraceptive use, family history of BC, delayed child birth and reduced duration of breast feeding have been identified as risk factors based on previous studies. Oral contraceptive use may induce the proliferation of breast cells, therefore increasing the risk of breast development (23). It has also been determined that a window of susceptibility to breast cancer exists, in the time period between puberty onset and first full-term pregnancy (15). The process of pregnancy transforms pubescent breast tissue into fully mature tissue that contains type- 4 lobules (24). The conclusion was that these factors are strongly associated with an increased risk of BC development (25). Hence, the combination of estrogen receptor status and plasma 
A

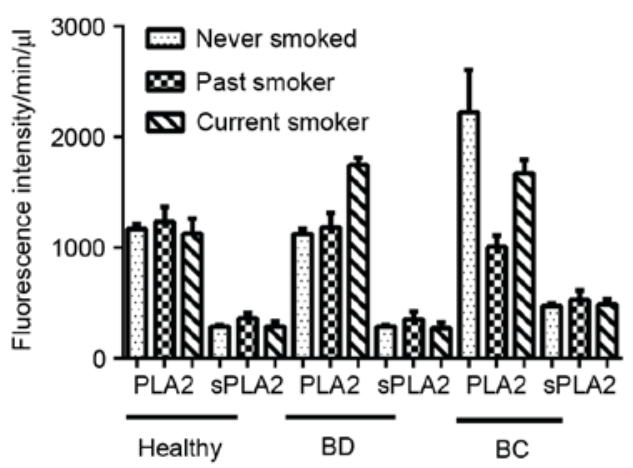

B

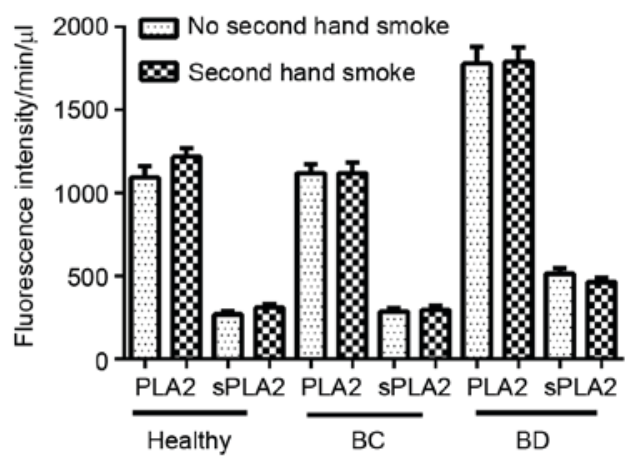

Figure 4. Effect of smoking status on PLA2 and sPLA2 activity. The smoking status of 81 healthy controls, 80 patients with benign disease and 169 patients with breast cancer were collected. (A) Effect of different smoking histories on PLA2 and sPLA2 activity. (B) Effect of exposure to secondhand smoke on PLA2 and sPLA2 activity. The majority of the patients in the present study were non-smokers. One-way analysis of variance was used to assess the differences between the three groups. No statistical significance was identified. PLA2, phospholipase A2; sPLA2, secretory phospholipase A2; BD, benign disease; BC, breast cancer $(\mathrm{P}<0.05$ was considered to indicate a statistically significant difference).

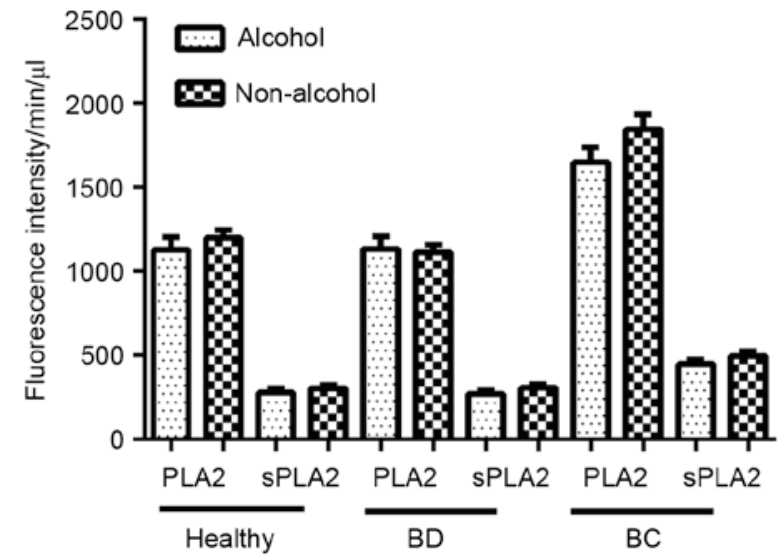

Figure 5. Effect of alcohol consumption on PLA2 and sPLA2 activity. Whether patients consumed alcohol or not was determined in 328 patients; 167 in patients with $\mathrm{BC}, 80$ in patients with $\mathrm{BD}$ and 81 in healthy controls. One-way analysis of variance was used to assess the differences in PLA2 and sPLA2 fluorescence intensity among between the three groups. No statistical significance was identified. PLA2, phospholipase A2; sPLA2, secretory phospholipase A2; $\mathrm{BD}$, benign disease; $\mathrm{BC}$, breast cancer $(\mathrm{P}<0.05$ was considered to indicate a statistically significant difference).

PLA2 activity may serve as a biomarker to predict the survival of patients with BC.

A lack of screening for $\mathrm{BC}$ may be the reason for increased rates of mortality among patients with BC (26). A previous study identified that there has been a large increase in metastatic breast malignancy (to the bone, brain and lungs) in young women at the time of diagnosis (15). Therefore, a more effective and convenient screening method is required to reduce mortality and prolong overall survival. Although image-based detection methods, including infrared ray scanning, B ultrasound and mammography are effective for the early detection of $\mathrm{BC}$, it has been determined that $\sim 40 \%$ of $\mathrm{BC}$ cases remain undiagnosed (27). In addition, certain modern imaging techniques, including breast molybdenum target mammography, produce false positive results, are expensive, inconvenient and require painful patient examinations $(13,28)$. Early detection of BC has contributed to a $3 \%$ annual decline in patient mortality rate (12).

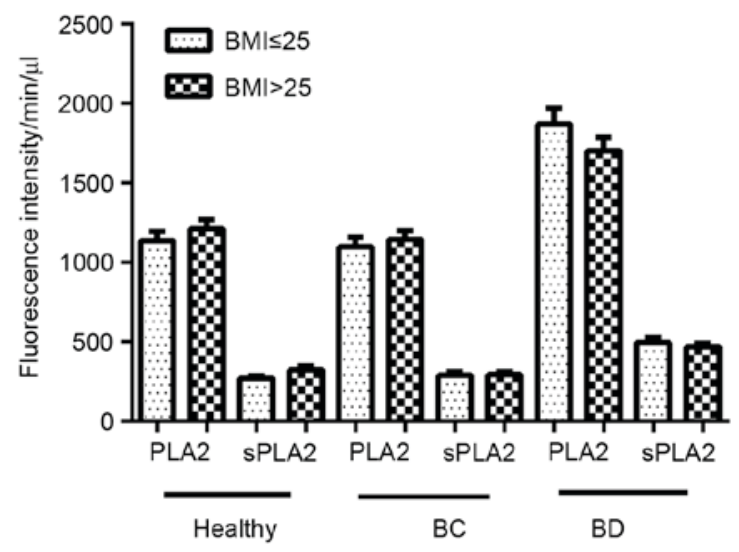

Figure 6. Effect of BMI on PLA2 and sPLA2 activity. BMI records were made available for 324 participants, including 163 patients with BC, 80 patients with $\mathrm{BD}$ and 81 healthy controls. Each group was then sub-grouped in to $\mathrm{BMI} \leq 25$ and $\mathrm{BMI}>25$ groups. And then One-way analysis of variance was used to assess differences in PLA2 and sPLA2 fluorescence intensity. No statistical significance was identified. PLA2, phospholipase A2; sPLA2, secretory phospholipase A2; BMI, body mass index; $\mathrm{BC}$, breast cancer; $\mathrm{BD}$, benign disease $(\mathrm{P}<0.05$ was considered to indicate a statistically significant difference).

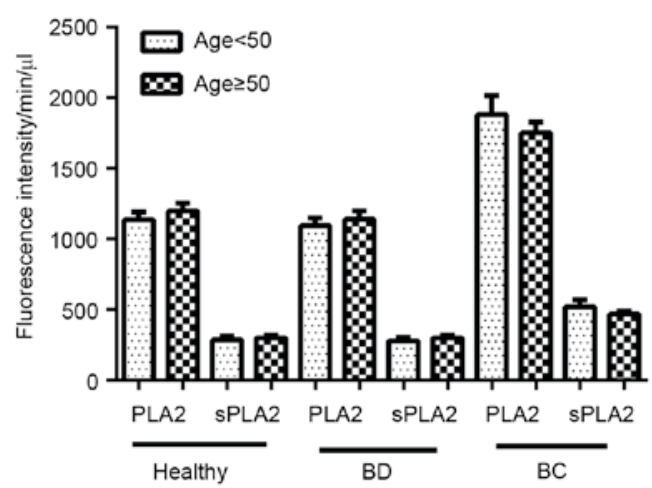

Figure 7. Effect of age on PLA2 and sPLA2 activity. The ages of all 330 patients included in the present study were obtained (169 patients with $\mathrm{BC}, 80$ patients with $\mathrm{BD}$ and 81 healthy controls). An age of 50 years was used as a standard to assess the age distribution among the three groups due to menopausal status. No significant differences in PLA2 and SPLA2 activities were found using one way ANOVA. PLA2, phospholipase A2; sPLA2, secretory phospholipase $\mathrm{A} 2 ; \mathrm{BC}$, breast cancer; $\mathrm{BD}$, benign disease $(\mathrm{P}<0.05$ was considered to indicate a statistically significant difference). 
The development of non-invasive screening techniques for $\mathrm{BC}$ is required. Molecular diagnostic approaches are often non-invasive and may serve to improve the specificity and sensitivity of BC screening (15). It has been demonstrated that serological biomarkers are effective in the early diagnosis of various types of cancer, and are also cost-effective and convenient $(19,29)$. Furthermore, serological biomarkers may functionally detect cancers at early stages and elucidate the molecular mechanisms that underlie the development of cancer, potentially leading to the establishment of novel therapeutic strategies $(13,29)$.

The present study determined that measuring PLA2 activity using a fluorescent plate reader is convenient and is able to be developed into an automated test. Furthermore, only a small quantity of plasma $(1-10 \mathrm{ml})$ is required to perform the test, the results of which are obtained in 1-2 h. Additional biomarkers or more specific modalities, including imaging techniques, may then be implemented for further examinations. An additional advantage to the PLA2 activity test is its reproducibility, stability and automated mechanism. A previous study established that blood biomarkers are sensitive to the handling, processing and storage of patient samples (13). For example, samples are particularly sensitive to freeze-thaw cycles. This explains why various sensitive and specific biomarkers discovered using proteomics are not utilized in clinical settings. The present study compared the CV of PLA2 and sPLA2 following experimental repeats and the induction of freeze-thaw cycling. The results demonstrated that PLA2 activities are highly consistent and independent of certain confounding factors, including freeze-thaw cycling, making them more likely to be effective biomarkers.

The activity of PLA2 may be restricted in patients with benign inflammatory diseases as these enzymes may be elevated owing to their involvement in inflammation. Inflammation is a response to internal and external environmental stimuli that functions to eliminate foreign agents and restore the physiology of normal tissue. However, when inflammation becomes chronic, it can result in the development of cancer $(30,31)$. Inflammation following viral infection can drive the progression of cancer (32). Epidemiological data has indicated that $>25 \%$ of various types of cancer are associated with chronic infection and inflammation (29). It has been demonstrated that the administration of non-steroidal anti-inflammatory drugs is associated with a reduction in the risk of developing various types of cancer, including BC (33). A previous study identified that SPLA2 regulates the activation of Ras and extracellular signal-regulated kinase and induces the phosphorylation of the epidermal growth factor receptor via a protein kinase C-dependent pathway, which may lead to a poor patient prognosis in an inflammatory microenvironment (34).

In conclusion, the results of the present study demonstrated that elevated PLA2 and sPLA2 activities were detected in patients with BC, particularly at late disease stages. Following the analysis of confounding factors, it was determined that disease type was as an independent factor, exhibiting no association with age, smoking, alcohol or BMI. The results indicated that plasma PLA2 activity might be a potential prognostic biomarker for patients with BC. Elevated PLA2 and SPLA2 activities may serve a vital role in breast carcinogenesis and could represent a novel therapeutic target for patients with $\mathrm{BC}$.

\section{Acknowledgements}

The present study was supported by the National Natural Science Foundation of China (grant no. 81502295) and Clinical Innovation Funds of The First Affiliated Hospital of XJTU (grant no. 14YB11).

\section{References}

1. Siegel RL, Miller KD and Jemal A: Cancer statistics, 2016. CA Cancer J Clin 66: 7-30, 2016.

2. Hassan LM, Mahmoud N, Miller AB, Iraj H, Mohsen M, Majid J, Reza SM and Mojgan M: Evaluation of effect of self-examination and physical examination on breast cancer. Breast 24: 487-490, 2015.

3. Curigliano G, Spitaleri G, Dettori M, Locatelli M, Scarano E and Goldhirsch A: Vaccine immunotherapy in breast cancer treatment: Promising, but still early. Expert Rev Anticancer Ther 7: 1225-1241, 2007.

4. Li SY, Li R, Chen YL, Xiong LK, Wang HL, Rong L and Luo RC: Aberrant PTPRO methylation in tumor tissues as a potential biomarker that predicts clinical outcomes in breast cancer patients. BMC Genet 15: 67, 2014.

5. Wang X, Huang CJ, Yu GZ, Wang JJ, Wang R, Li YM and Wu Q: Expression of group IIA phospholipase A2 is an independent predictor of favorable outcome for patients with gastric cancer. Hum Pathol 44: 2020-2027, 2013.

6. Li S, Zhao X, Wu Z, Li Y, Zhu L, Cui B, Dong X, Tian S, Hu F and Zhao Y: Polymorphisms in arachidonic acid metabolism-related genes and the risk and prognosis of colorectal cancer. Fam Cancer 12: 755-765, 2013.

7. Mirtti T, Laine VJ, Hiekkanen H, Hurme S, Rowe O, Nevalainen TJ, Kallajoki M and Alanen K: Group IIA phospholipase A as a prognostic marker in prostate cancer: Relevance to clinicopathological variables and disease-specific mortality. APMIS 117: 151-161, 2009.

8. Menschikowski M, Hagelgans A, Nacke B, Jandeck C, Mareninova OA, Asatryan L and Siegert G: Epigenetic control of group $\mathrm{V}$ phospholipase $\mathrm{A} 2$ expression in human malignant cells. Tumour Biol 37: 8097-8105, 2016.

9. Quach ND, Arnold RD and Cummings BS: Secretory phospholipase A2 enzymes as pharmacological targets for treatment of disease. Biochem Pharmacol 90: 338-348, 2014.

10. Jin Y, Yang F and Du L: Nanoassemblies containing a fluorouracil/zidovudine glyceryl prodrug with phospholipase A2-triggered drug release for cancer treatment. Colloids Surf B Biointerfaces 112: 421-428, 2013.

11. Zhu C, Sun Z, Li C, Guo R, Li L, Jin L, Wan R and Li S: Urocortin affects migration of hepatic cancer cell lines via differential regulation of cPLA2 and iPLA2. Cell Signal 26: 1125-1134, 2014.

12. Mannello F, Qin W, Zhu W, Fabbri L, Tonti GA and Sauter ER: Nipple aspirate fluids from women with breast cancer contain increased levels of group IIa secretory phospholipase A2. Breast Cancer Res Treat 111: 209-218, 2008.

13. Kondo T: Inconvenient truth: Cancer biomarker development by using proteomics. Biochim Biophys Acta 1844: 861-865, 2014.

14. Cai Q, Zhao Z, Antalis C, Yan L, Del Priore G, Hamed AH, Stehman FB, Schilder JM and Xu Y: Elevated and secreted phospholipase $\mathrm{A}_{2}$ activities as new potential therapeutic targets in human epithelial ovarian cancer. FASEB J 26: 3306-3320, 2012.

15. Schneider AP II, Zainer CM, Kubat CK, Mullen NK and Windisch AK: The breast cancer epidemic: 10 facts. Linacre Q 81: 244-277, 2014.

16. Lanfranchi AE and Fagan P: Breast cancer and induced abortion: A comprehensive review of breast development and pathophysiology, the epidemiologic literature, and proposal for creation of databanks to elucidate all breast cancer risk factors. Issues Law Med 29: 3-133, 2014.

17. Linos E, Spanos D, Rosner BA, Linos K, Hesketh T, Qu JD, Gao YT, Zheng W and Colditz GA: Effects of reproductive and demographic changes on breast cancer incidence in China: A modeling analysis. J Natl Cancer Inst 100: 1352-1360, 2008. 
18. World Medical Association: World medical association declaration of helsinki: Ethical principles for medical research involving human subjects. JAMA 310: 2191-2194, 2013.

19. Cai H, Chiorean EG, Chiorean MV, Rex DK, Robb BW, Hahn NM, Liu Z, Loehrer PJ, Harrison ML and Xu Y: Elevated phospholipase A2 activities in plasma samples from multiple cancers. PLoS One 8: e57081, 2013.

20. Sobin LH and Wittekind C: TNM classification of malignant tumors. International Union Against Cancer (UICC), 5th edition, pp54-58, 1997.

21. Buhmeida A, Bendardaf R, Hilska M, Laine J, Collan Y, Laato M, Syrjänen K and Pyrhönen S: PLA2 (group IIA phospholipase A2) as a prognostic determinant in stage II colorectal carcinoma. Ann Oncol 20: 1230-1235, 2009.

22. Yamashita S, Yamashita J, Sakamoto K, Inada K, Nakashima Y, Murata K, Saishoji T, Nomura K and Ogawa M: Increased expression of membrane-associated phospholipase A2 shows malignant potential of human breast cancer cells. Cancer 71: 3058-3064, 1993

23. Lovett JL, Chima MA, Wexler JK, Arslanian KJ, Friedman AB, Yousif CB and Strassmann BI: Oral contraceptives cause evolutionarily novel increases in hormone exposure: A risk factor for breast cancer. Evol Med Public Health 2017: 97-108, 2017.

24. Russo J, Moral R, Balogh GA, Mailo D and Russo IH: The protective role of pregnancy in breast cancer. Breast Cancer Res 7: 131-142, 2005.

25. Kamińska M, Ciszewski T, Łopacka-Szatan K, Miotła P and Starosławska E: Breast cancer risk factors. Prz Menopauzalny 14: 196-202, 2015

26. Webb ML, Cady B, Michaelson JS, Bush DM, Calvillo KZ, Kopans DB and Smith BL: A failure analysis of invasive breast cancer: Most deaths from disease occur in women not regularly screened. Cancer 120: 2839-2846, 2014.
27. Howell A: The emerging breast cancer epidemic: Early diagnosis and treatment. Breast Cancer Res 12 (Suppl 4): S10, 2010.

28. Killelea BK, Long JB, Chagpar AB, Ma X, Wang R, Ross JS and Gross CP: Evolution of breast cancer screening in the Medicare population: Clinical and economic implications. J Natl Cancer Inst 106: pii: dju159, 2014.

29. Indovina P, Marcelli E, Pentimalli F, Tanganelli P, Tarro G and Giordano A: Mass spectrometry-based proteomics: The road to lung cancer biomarker discovery. Mass Spectrom Rev 32: 129-142, 2013.

30. Vendramini-Costa DB and Carvalho JE: Molecular link mechanisms between inflammation and cancer. Curr Pharm Des 18: 3831-3852, 2012

31. Gurel B, Lucia MS, Thompson IM Jr, Goodman PJ, Tangen CM, Kristal AR, Parnes HL, Hoque A, Lippman SM, Sutcliffe S, et al: Chronic inflammation in benign prostate tissue is associated with high-grade prostate cancer in the placebo arm of the prostate cancer prevention trial. Cancer Epidemiol Biomarkers Prev 23: 847-856, 2014.

32. Deivendran S, Marzook KH and Radhakrishna Pillai M: The role of inflammation in cervical cancer. Adv Exp Med Biol 816: 377-399, 2014.

33. Liu Q, Tan Q, Zheng Y, Chen K, Qian C, Li N, Wang Q and Cao X: Blockade of Fas signaling in breast cancer cells suppresses tumor growth and metastasis via disruption of Fas signaling-initiated cancer-related inflammation. J Biol Chem 289: 11522-11535, 2014.

34. Hernández M, Martín R, García-Cubillas MD, Maeso-Hernández P and Nieto ML: Secreted PLA2 induces proliferation in astrocytoma through the EGF receptor: Another inflammation-cancer link. Neuro Oncol 12: 1014-1023, 2010 . 